\title{
ON SUPERCRITICAL SOBOLEV TYPE INEQUALITIES AND RELATED ELLIPTIC EQUATIONS
}

\author{
JOÃO MARCOS DO Ó, BERNHARD RUF, AND PEDRO UBILLA
}

\begin{abstract}
Sobolev type embeddings for radial functions into variable exponent Lebesgue spaces are studied. In particular, the following inequality is proved:

Let $B \subset \mathbb{R}^{N}, N \geq 3$, be the unit ball, and let $H_{0 \text {,rad }}^{1}(B)$ denote the first order Sobolev space of radial functions, and $2^{*}=2 N /(N-2)$ the corresponding critical Sobolev embeddding exponent. Let $r=|x|$, and $p(r)=2^{*}+r^{\alpha}$, with $\alpha>0$; then

$$
\sup \left\{\int_{B}|u|^{p(r)} d x \mid u \in H_{0, \mathrm{rad}}^{1}(B),\|\nabla u\|_{2}=1\right\}<+\infty .
$$

We point out that the growth of $p(r)$ is strictly larger than $2^{*}$, except in the origin.

Furthermore, we show that for $p(r)=2^{*}+r^{\alpha}$, with $0<\alpha<\min \left\{\frac{N}{2} ; N-\right.$ $2\}$, the supremum in (0.1) is attained.

Finally, we prove that associated elliptic equations admit nontrivial radial solutions. This is somewhat surprising since the nonlinearities have strictly supercritical growth except in the origin.
\end{abstract}

\section{INTRODUCTION AND MAIN RESULTS}

Let $\Omega \subset \mathbb{R}^{N}$ denote a bounded domain in $\mathbb{R}^{N}, N \geq 3$. The Sobolev embeddings yield explicit (critical) exponents for embeddings into Lebesgue $L^{p_{-}}$ spaces, $W^{1,2}(\Omega) \subset L^{2^{*}}(\Omega)$, which are optimal within the class of $L^{p}$ spaces. Extending to the class of "rearrangement invariant" (r.i.) Banach spaces, the Sobolev embeddings may be slightly improved by going to Lorentz spaces $L^{p, q}(\Omega)$ (see Peetre [9] and Tartar [15]); Lorentz spaces $L^{p, q}$ are scales of rearrangement invariant interpolation spaces between Lebesgue spaces $L^{p}$. Indeed, one has

$$
W^{1,2}(\Omega) \subset L^{2^{*}, 2}(\Omega)
$$

It is inherent to the definiton of r.i. Banach spaces that the inequalities remain valid under symmetrization, and hence it is sufficient to prove the respective inequalities in the radial context.

2000 Mathematics Subject Classification. 35J20, 35J25, 35J50.

Key words and phrases. Critical Sobolev exponent, supercritical growth, supercritical elliptic problems,

Research supported in part by INCTmat/MCT/Brazil, CNPq, CAPES/Brazil and FONDECYT 1120524. 
In this article we consider inequalities and related embeddings into non rearrangement inavariant Banach spaces. In fact, our targes spaces are variable exponent Lebesgue spaces (see [8]), which have received wide attention in recent years. Since symmetrization cannot be applied in this situation, and in order to obtain optimal results, we are lead to restrict the spaces to functions which are adapted to the variable exponents. In particular, for radially symmetric variable exponents, it is natural to restrict to spaces of radially symmetric functions. On the other hand, we will see that this restriction allows for a considerable gain in growth.

In particular, we will prove the following inequality: Let $B \subset \mathbb{R}^{N}$ denote the unit ball, $r=|x|$ and let $H_{0, \text { rad }}^{1}(B)$ be the first order Sobolev space of radial functions, and $2^{*}=2 N /(N-2)$ the corresponding critical Sobolev embeddding exponent.

Theorem 1.1. Let $p(r)=2^{*}+r^{\alpha}$ and $\alpha>0$; then

$$
\sup \left\{\int_{B}|u(x)|^{p(r)} d x: u \in H_{0, \operatorname{rad}}^{1}(B),\|\nabla u\|_{2}=1\right\}<+\infty .
$$

We emphasize that the variable exponent $p(r)$ has critical Sobolev growth only in the origin, and is strictly supercritical everywhere else. Furthermore, if $0<\alpha<1$, then the derivative of $p(r)$ in the origin is $+\infty$.

As a consequence of Theorem 1.1, we have the following embedding of the subspace of radial functions in $H_{0}^{1}(B)$ into a not rearrangement invariant Lebesgue space with variable exponent:

Corollary 1.2. The following embedding is continuous:

$$
H_{0, \mathrm{rad}}^{1}(B) \hookrightarrow L_{p(r)}(B),
$$

where $L_{p(r)}$ is defined as follows (see e.g. [8])

$$
L_{p(r)}(B):=\left\{u: B \rightarrow \mathbb{R} \text { measurable }: \int_{B}|u(x)|^{p(r)} d x<\infty\right\}
$$

with norm

$$
\|u\|_{p(r)}=\inf \left\{\lambda>0, \int_{B}\left|\frac{u(x)}{\lambda}\right|^{p(r)} \mathrm{d} x \leq 1\right\} .
$$

Next, let $\Sigma_{N}$ denote the following best constant of Sobolev type

$$
\Sigma_{N}:=\sup _{\left\{u \in H_{0, \text { rad }}^{1}(B):\|\nabla u\|_{2}=1\right\}} \int_{B}|u(x)|^{2^{*}} d x
$$

and consider the analogous constant in the variable exponent space $L_{p(r)}$

$$
\mathcal{U}_{N}:=\sup _{\left\{u \in H_{0, \mathrm{rad}}^{1}(B):\|\nabla u\|_{2}=1\right\}} \int_{B}|u(x)|^{p(r)} d x .
$$

We will show the following theorem 
Theorem 1.3. If $p(r)=2^{*}+r^{\alpha}$ and

$$
0<\alpha<\min \{N / 2, N-2\}
$$

then:

$$
\mathcal{U}_{N}>\Sigma_{N}
$$

The restriction (1.5) says that $p(r)$ may not be too flat near the origin for (1.6) to hold.

It is well-kown that the supremum $\Sigma_{N}$ in (1.3) is not attained whenever $\Omega \neq \mathbb{R}^{N}$. The following theorem shows that $\mathcal{U}_{N}$ is attained if it is larger than $\Sigma_{N}$

Theorem 1.4. If $\mathcal{U}_{N}>\Sigma_{N}$, then the supremum $\mathcal{U}_{N}$ is attained.

We recall the seminal work of Brezis-Nirenberg [2] who proved that adding a suitable lower order perturbation to the critical Sobolev exponent functional leads to a conclusion like (1.6), and then that the corresponding supremum is attained. Here we have an analogous result, but with a very different growth function: the variable exponent function has a strictly supercritical growth everywhere except in the origin, that is, we have a "supercritical perturbation" of the critical exponent $2^{*}$.

Next, we consider a related elliptic equation with a supercritical nonlinearity.

Theorem 1.5. Let $p(r)=2^{*}+r^{\alpha}$, with $\alpha$ satisfying condition (1.5). Then the following boundary value problem has a nontrivial radial solution:

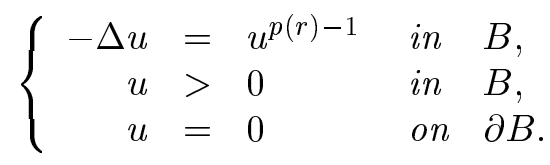

Note that the nonlinearity $u^{2^{*}-1+r^{\alpha}}$ has a highly supercritical growth (expect in the origin where the growth is critical). Thus, the existence of a solution is quite surprising: recall that by Pohozaev's identity [10] equation (1.7) has no non-trivial solution if $p(r)=2^{*}+c$, with $c \geq 0$, while we obtain the existence of a solution for any $p(r)$ of the form $p(r)=2^{*}+r^{\alpha}$. For existence results concerning equations with critical growth and with conditions on the domain, we refer to the results of Bahri-Coron [1] and Coron [3], while for equations with slightly supercritical growth we recall the articles of del Pino [4], del Pino-Wei [7], see also [6], [5], and for other types of solutions in supercritical equations, [11], [12].

The proof of Theorem 1.5 follows the ideas of [2]: using the mountain-pass theorem one constructs a minimax level for the functional corresponding to equation (1.7). Using the structure of the nonlinearity it is then shown that this level lies below the non-compactness level of the functional, and is thus a critical value. 
Plan of the Paper. In Sect. 2, we prove a more general version of Theorem 1.1. In Sect. 3, we prove Theorem 1.3. In Sect. 4, we show that the supremum $\mathcal{U}_{N}$ defined in (1.4) is attained. In Sect. 5, we show the existence of nontrivial solution of (1.7), which as we already emphasized is a semilinear elliptic problem involving highly supercritical growth.

\section{The INEQUALITY}

In this section we state a more general version of Theorem 1.1 and give the proof. Let $f:[0,1) \rightarrow \mathbb{R}^{+}$be a continuous function satisfying the following conditions:

$\left(f_{1}\right) f(0)=0$ and $f(r)>0$ for all $r>0$;

$\left(f_{2}\right) f(r) \leq \frac{c}{|\log r|^{\beta}}$ for some $\beta>2$, for $r$ near 0 ;

$\left(f_{3}\right) f(r) \leq \frac{c}{|1-r|}$, for $r$ near 1.

We recall the following facts: Let $S_{N}$ be the best constant in the Sobolev embedding $H^{1}\left(\mathbb{R}^{N}\right) \hookrightarrow L^{2^{*}}\left(\mathbb{R}^{N}\right)$ with $2^{*}=2 N /(N-2)$, that is

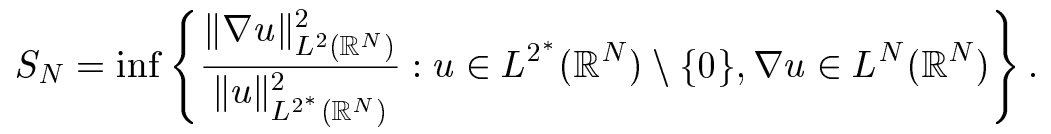

It is well-known that the infimum $S_{N}$ is achieved. Moreover, for every open subset $\Omega$ of $\mathbb{R}^{N}$, let

$$
S_{N}(\Omega):=\inf \left\{\|\nabla u\|_{2}^{2}: u \in H_{0}^{1}(\Omega),\|u\|_{2^{*}}=1\right\} .
$$

It is known that $S_{N}(\Omega)=S_{N}$, and that $S_{N}(\Omega)$ is never achieved except when $\Omega=\mathbb{R}^{N}$.

We will use throughout the paper the notation $|x|=r$.

We now state and prove the following general inequality:

Theorem 2.1. Assume that $f \in C\left([0,1), \mathbb{R}^{+}\right)$satisfies $\left(f_{1}\right)-\left(f_{3}\right)$, and let $p(r)=2^{*}+f(r)$. Then

$$
\sup \left\{\int_{B}|u(x)|^{p(r)} \mathrm{d} x: u \in H_{0, \mathrm{rad}}^{1}(B),\|\nabla u\|_{2}=1\right\}<+\infty .
$$

Note that $f(r)=r^{\alpha}, \alpha>0$, satisfies $\left(f_{1}\right)-\left(f_{3}\right)$, and hence Theorem 2.1 implies Theorem 1.1.

We are going to use the following versions of the "radial lemma" (see [16]).

Lemma 2.2. Let $u \in H_{0, \mathrm{rad}}^{1}(B)$. Then

$$
|u(r)| \leq \frac{1}{(N-2)^{1 / 2}} \frac{\|\nabla u\|_{2}}{r^{(N-2) / 2}}
$$


and

$$
|u(r)| \leq \frac{(1-r)^{1 / 2}}{r^{(N-2) / 2}}\|\nabla u\|_{2}
$$

Proof. If $u \in H_{0, \text { rad }}^{1}(B)$, then

$$
\begin{aligned}
|u(r)| & \leq\left|\int_{r}^{1} u^{\prime}(s) \mathrm{d} s\right| \leq \int_{r}^{1}\left|u^{\prime}(s) s^{(N-1) / 2} \frac{1}{s^{(N-1) / 2}}\right| \mathrm{d} s \\
& \leq\|\nabla u\|_{L^{2}}\left\|\frac{1}{s^{(N-1) / 2}}\right\|_{L^{2}([r, 1])}=\|\nabla u\|_{L^{2}}\left(\frac{r^{2-N}-1}{N-2}\right)^{1 / 2}
\end{aligned}
$$

which yields (2.3). To obtain (2.4), note that we can rewrite

$$
\begin{aligned}
\left(\frac{r^{2-N}-1}{N-2}\right)^{1 / 2} & =\frac{1}{\sqrt{N-2}}\left(\frac{1}{r^{N-2}}-1\right)^{1 / 2} \\
& =\frac{1}{\sqrt{N-2}}\left(\frac{1-r^{N-2}}{r^{N-2}}\right)^{1 / 2} \\
& =\frac{1}{\sqrt{N-2}} \frac{1}{r^{(N-2) / 2}}\left(1-r^{N-2}\right)^{1 / 2} \\
& =\frac{1}{\sqrt{N-2}} \frac{1}{r^{(N-2) / 2}}\left((1-r)\left(1+r+\cdots+r^{N-3}\right)\right)^{1 / 2} \\
& \leq \frac{1}{r^{(N-2) / 2}}(1-r)^{1 / 2}
\end{aligned}
$$

which together with (2.5) establishes (2.4).

2.1. Proof of Theorem 2.1. For $u \in H_{0, \text { rad }}^{1}(B)$ with $\|\nabla u\|_{2}=1$, we can write (here $\omega_{\mathrm{N}-1}$ is the surface area of the unit sphere in $\mathbb{R}^{N}$ )

$\frac{1}{\omega_{N-1}} \int_{B}|u(x)|^{2^{*}+f(r)} d x=\int_{0}^{\rho}|u(r)|^{2^{*}+f(r)} r^{N-1} d x+\int_{\rho}^{1}|u(r)|^{2^{*}+f(r)} r^{N-1} d r$,

where $\rho$ will be determined later. We shall estimate each of these two terms separately.

$$
\begin{aligned}
\int_{0}^{\rho}|u(r)|^{2^{*}+f(r)} r^{N-1} d x & =\int_{0}^{\rho}|u(r)|^{2^{*}}\left(|u(r)|^{f(r)}-1\right) r^{N-1} d x+\int_{0}^{\rho}|u(r)|^{2^{*}} r^{N-1} d x \\
& \leq \int_{0}^{\rho}|u(r)|^{2^{*}}\left(|u(r)|^{f(r)}-1\right) r^{N-1} d x+\Sigma_{N} .
\end{aligned}
$$

By the Radial Lemma 2.2 for $u \in H_{0, \text { rad }}^{1}\left(B_{1}\right)$ with $\|\nabla u\|_{2}=1$ we can write

$$
|u(r)| \leq \frac{(1-r)^{1 / 2}}{r^{(N-2) / 2}} \leq \frac{1}{r^{(N-2) / 2}}
$$


which implies

$$
\begin{aligned}
\int_{0}^{\rho}|u(r)|^{2^{*}} & \left.|u(r)|^{f(r)}-1\right) r^{N-1} d x \\
& \leq \int_{0}^{\rho}\left(\frac{1}{r^{(N-2) / 2}}\right)^{2 N /(N-2)}\left[\left(\frac{1}{r^{(N-2) / 2}}\right)^{f(r)}-1\right] r^{N-1} d r \\
& \leq \int_{0}^{\rho} \frac{1}{r}\left[\exp \left(f(r) \log \frac{1}{r^{(N-2) / 2}}\right)-1\right] d r \\
& \leq \int_{0}^{\rho} \frac{1}{r}\left[\exp \left(\frac{N-2}{2} f(r)|\log r|\right)-1\right] d r \\
& \leq d \frac{N-2}{2} \int_{0}^{\rho} f(r) \frac{|\log r|}{r} d r
\end{aligned}
$$

where in the estimates above we have used that given $d>1$ there exists $\rho=\rho(d)>0$ such that

$$
e^{g(s)}-1 \leq d g(s), \forall s \in(0, \rho), \text { provided that } g(s) \rightarrow 0 \text {, as } s \rightarrow 0,
$$

which is the case for $g(r)=\frac{N-2}{2} f(r)|\log r|$ by assumption $\left(f_{2}\right)$. Therefore, in order to obtain

$$
\int_{0}^{\rho}|u(r)|^{2^{*}}\left(|u(r)|^{f(r)}-1\right) r^{N-1} d x<\infty
$$

we have to impose the condition

$$
\int_{0}^{\rho} f(r) \frac{|\log r|}{r} d r<\infty
$$

Notice that (2.8) holds if we assume condition $\left(f_{2}\right)$.

To estimate the second integral in (2.6), using again (2.7) and assumption $\left(f_{3}\right)$, we can write

$$
\begin{aligned}
\int_{\rho}^{1}|u(r)|^{2^{*}+f(r)} r^{N-1} d r & \leq \int_{\rho}^{1}\left(\frac{1}{r^{(N-2) / 2}}\right)^{2^{*}+f(r)} r^{N-1} d r \\
& =\int_{\rho}^{1} \frac{1}{r^{1+\frac{N-2}{2} f(r)}} d r \\
& =-\int_{1-\rho}^{0} \frac{1}{(1-s)^{1+\frac{N-2}{2} f(1-s)}} d s \\
& \leq \int_{0}^{1-\rho} \frac{1}{(1-s)^{1+\frac{N-2}{2} \frac{c}{s}}} d s \\
& =\int_{0}^{1-\rho} \frac{1}{e^{\left(1+\frac{N-2}{2} \frac{c}{s}\right) \log (1-s)}} \mathrm{d} s
\end{aligned}
$$

Since $e^{\left(1+\frac{N-2}{2} \frac{c}{s}\right) \log (1-s)} \sim e^{-\left(1+\frac{N-2}{2} \frac{c}{s}\right) s}$ for $s$ near 0 (i.e. for $r$ near 1 ), the integral is finite. 
Example 2.3. For $r$ near 0 , consider

1) $f(r)=\frac{1}{|\log r|^{2+\delta}}$, for $\delta>0$.

2) $f(r)=r^{\alpha}|\log r|^{\eta}$, for $\alpha>0$ and $\eta \geq 0$.

For $r$ near 1 , consider $f(r)=1 /(1-r)$.

2.2. Proof of Corollary 1.2. Consider the variable exponent Lebesgue space

$$
L^{2^{*}+f(r)}(B):=\left\{u: B \rightarrow \mathbb{R} \text { measurable }: \int_{B}|u(x)|^{2^{*}+f(r)} d x<\infty\right\}
$$

endowed with the norm

$$
\|u\|_{2^{*}+f(r)}:=\inf \left\{\lambda>0: \int_{B}\left|\frac{u(x)}{\lambda}\right|^{2^{*}+f(r)} d x \leq 1\right\} .
$$

Assuming that $u \in H_{0, \text { rad }}^{1}(B)$ with $\|\nabla u\|_{2}=1$, we have to prove that $\|u\|_{2^{*}+f(r)} \leq C$. Using Theorem 2.1 we know that

$$
\int_{B}|u(x)|^{2^{*}+f(r)} d x=C<\infty
$$

For $\lambda>1$ we can estimate

$$
\int_{B}\left|\frac{u(x)}{\lambda}\right|^{2^{*}+f(r)} d x \leq \int_{B} \frac{|u(x)|^{2^{*}+f(r)}}{\lambda^{2^{*}}} d x \leq \frac{C}{\lambda^{2^{*}}} \leq 1
$$

if $\lambda=\lambda_{u}$ is sufficiently large. Then, $\|u\|_{2^{*}+f(r)} \leq \lambda_{u}$.

\section{The Supremum $\mathcal{U}_{N}$}

From now on we assume that

$$
f(r)=r^{\alpha}, \text { with } 0<\alpha<\min \{N / 2, N-2\} .
$$

3.1. Proof of Theorem 1.3. Let us denote with

$$
\begin{aligned}
u_{\varepsilon}^{*}(r) & =C_{N} \frac{\varepsilon^{\frac{N-2}{2}}}{\left(\varepsilon^{2}+r^{2}\right)^{\frac{N-2}{2}}}, \quad \varepsilon>0 \\
C_{N} & :=(N(N-2))^{\frac{N-2}{2}}
\end{aligned}
$$

the standard Sobolev instantons, see [14], [13], which satisfy the equation

$$
-\Delta u=u^{2^{*}-1}, \text { on } \mathbb{R}^{N}
$$

and for which

$$
\int_{\mathbb{R}^{N}}\left|\nabla u_{\varepsilon}^{*}(x)\right|^{2} d x=S_{N}^{N / 2} \text { and } \int_{\mathbb{R}^{N}}\left|u_{\varepsilon}^{*}(x)\right|^{2^{*}} d x=S_{N}^{N / 2}
$$


where $S_{N}$ is as in (2.1). Let $\eta$ be a suitable cut-off function; it is then well-known (see [2]) that

$$
\begin{gathered}
\left\|\nabla\left(\eta u_{\varepsilon}^{*}\right)\right\|_{2}^{2}=S_{N}^{N / 2}+O\left(\varepsilon^{N-2}\right), \\
\left\|\eta u_{\varepsilon}^{*}\right\|_{2^{*}}^{2^{*}}=S_{N}^{N / 2}+O\left(\varepsilon^{N}\right) .
\end{gathered}
$$

Let now $B_{N}:=1 / S_{N}^{N / 4}$, and

$$
u_{\varepsilon}(r):=B_{N} \eta(r) u_{\varepsilon}^{*}(r)=A_{N} \eta(r) \frac{\varepsilon^{\frac{N-2}{2}}}{\left(\varepsilon^{2}+r^{2}\right)^{\frac{N-2}{2}}}
$$

with $A_{N}:=B_{N} C_{N}($ see $(3.2))$, so that

$$
\left\|\nabla u_{\varepsilon}\right\|_{L^{2}}=1+O\left(\varepsilon^{N-2}\right)
$$

and

$$
\begin{aligned}
\int_{B}\left|u_{\varepsilon}(x)\right|^{2^{*}} d x & =\int_{B} B_{N}^{2^{*}}\left|\eta(x) u_{\varepsilon}^{*}(x)\right|^{2^{*}} d x \\
& =\frac{S_{N}^{N / 2}+O\left(\varepsilon^{N}\right)}{\left(S_{N}^{N / 4}\right)^{2^{*}}} \\
& =S_{N}^{-\frac{N}{N-2}}+O\left(\varepsilon^{N}\right) \\
& =: \Sigma_{N}+O\left(\varepsilon^{N}\right) .
\end{aligned}
$$

Finally, by (3.4) and (3.5) we have

$$
\int_{B}\left(\frac{\left|u_{\varepsilon}(x)\right|}{\left\|\nabla u_{\varepsilon}(x)\right\|_{2}}\right)^{2^{*}} d x=\Sigma_{N}+O\left(\varepsilon^{N-2}\right)
$$

To prove (1.6) we will show the following

Lemma 3.1. There exists a constant $C>0$ such that for all $\varepsilon>0$ small

$$
\int_{B}\left|u_{\varepsilon}(x)\right|^{2^{*}+r^{\alpha}} d x \geq \int_{B}\left|u_{\varepsilon}(x)\right|^{2^{*}} d x+C|\log \varepsilon| \varepsilon^{\alpha}+O\left(\varepsilon^{N / 2}\right)+O\left(\varepsilon^{N-2}\right) .
$$

Then, since by assumption $\alpha \leq \min \{N / 2, N-2\}$, we conclude by asymptotics that

$$
\begin{aligned}
\mathcal{U}_{N} & =\sup \left\{\int_{B}|u(x)|^{2^{*}+r^{\alpha}} d x,\|\nabla u\|_{2}=1\right\} \\
& \geq \int_{B}\left(\frac{\left|u_{\varepsilon}(x)\right|}{\left\|\nabla u_{\varepsilon}\right\|_{2}}\right)^{2^{*}+r^{\alpha}} d x=\int_{B}\left|u_{\varepsilon}(x)\right|^{2^{*}+r^{\alpha}} d x+O\left(\varepsilon^{N-2}\right) \\
& \geq \int_{B}\left|u_{\varepsilon}(x)\right|^{2^{*}} d x+C|\log \varepsilon| \varepsilon^{\alpha}+O\left(\varepsilon^{N / 2}\right)+O\left(\varepsilon^{N-2}\right) \\
& >\Sigma_{N}
\end{aligned}
$$

which concludes the proof of Theorem 1.3. 
Proof of Lemma 3.1: First observe that

$$
B_{N} u_{\varepsilon}^{*}(r) \leq 1 \text { if and only if } r \geq\left(A_{N}^{2 /(N-2)} \varepsilon-\varepsilon^{2}\right)^{1 / 2}=: a_{\varepsilon}
$$

(recall that $A_{N}=B_{N} C_{N}$ ). We write

$$
\frac{1}{\omega_{N-1}} \int_{B}\left|u_{\varepsilon}(x)\right|^{2^{*}+r^{\alpha}} d x=\int_{0}^{a_{\varepsilon}}\left|u_{\varepsilon}(r)\right|^{2^{*}+r^{\alpha}} r^{N-1} d r+\int_{a_{\varepsilon}}^{1}\left|u_{\varepsilon}(r)\right|^{2^{*}+r^{\alpha}} r^{N-1} d r .
$$

First, we estimate the integral

$$
\begin{aligned}
\int_{a_{\varepsilon}}^{1}\left|u_{\varepsilon}(r)\right|^{2^{*}+r^{\alpha}} r^{N-1} d r & =\int_{a_{\varepsilon}}^{1}\left|\eta(r) u_{\varepsilon}(r)^{*}\right|^{2^{*}+r^{\alpha}} r^{N-1} d r \\
& \leq \int_{a_{\varepsilon}}^{1}\left|u_{\varepsilon}^{*}(r)\right|^{2^{*}} r^{N-1} d r \\
& =\int_{a_{\varepsilon}}^{1} \frac{A_{N}^{2^{*}} \varepsilon^{N}}{\left(\varepsilon^{2}+r^{2}\right)^{N}} r^{N-1} d r \\
& \leq \int_{a_{\varepsilon}}^{1} \frac{A_{N}^{2^{*}} \varepsilon^{N}}{r^{2 N}} r^{N-1} d r \\
& =A_{N}^{2^{*}} \varepsilon^{N} \int_{a_{\varepsilon}}^{1} r^{-N-1} d r \\
& =\left.\frac{A_{N}^{2^{*}} \varepsilon^{N}}{N}\left(-r^{-N}\right)\right|_{a_{\varepsilon}} ^{1} \\
& =\frac{A_{N}^{2^{*}} \varepsilon^{N}}{N}\left(\left(A_{N}^{2 /(N-2)} \varepsilon-\varepsilon^{2}\right)^{-N / 2}-1\right) \\
& =O\left(\varepsilon^{N / 2}\right) .
\end{aligned}
$$

As seen above, one also has

$$
\int_{a_{\varepsilon}}^{1}\left|u_{\varepsilon}(r)\right|^{2^{*}} r^{N-1} d r=O\left(\varepsilon^{N / 2}\right)
$$

Hence, we get

$$
\begin{aligned}
& \int_{0}^{1}\left|u_{\varepsilon}(r)\right|^{2^{*}+r^{\alpha}} r^{N-1} d r \\
& =\int_{0}^{1}\left|u_{\varepsilon}(r)\right|^{2^{*}} r^{N-1} d r+\int_{0}^{1}\left(\left|u_{\varepsilon}(r)\right|^{2^{*}+r^{\alpha}}-\left|u_{\varepsilon}(r)\right|^{2^{*}}\right) r^{N-1} d r \\
= & \int_{0}^{1}\left|u_{\varepsilon}(r)\right|^{2^{*}} r^{N-1} d r+\int_{0}^{a_{\varepsilon}}\left(\left|u_{\varepsilon}(r)\right|^{2^{*}+r^{\alpha}}-\left|u_{\varepsilon}(r)\right|^{2^{*}}\right) r^{N-1} d r+O\left(\varepsilon^{N / 2}\right) \\
= & \int_{0}^{1}\left|u_{\varepsilon}(r)\right|^{2^{*}} r^{N-1} d r+\int_{0}^{a_{\varepsilon}}\left(\left|B_{N} \eta u_{\varepsilon}^{*}(r)\right|^{2^{*}+r^{\alpha}}-\left|B_{N} \eta u_{\varepsilon}^{*}(r)\right|^{2^{*}}\right) r^{N-1} d r+O\left(\varepsilon^{N / 2}\right) \\
\geq & \int_{0}^{1}\left|u_{\varepsilon}(r)\right|^{2^{*}} r^{N-1} d r+\int_{0}^{\varepsilon}\left(\left|B_{N} u_{\varepsilon}^{*}(r)\right|^{2^{*}+r^{\alpha}}-\left|B_{N} u_{\varepsilon}^{*}(r)\right|^{2^{*}}\right) r^{N-1} d r+O\left(\varepsilon^{N / 2}\right),
\end{aligned}
$$


since $B_{N} u_{\varepsilon}^{*} \geq 1$ on the interval $\left[\varepsilon, a_{\varepsilon}\right]$. Set

$$
I_{1, \varepsilon}:=\int_{0}^{\varepsilon}\left|B_{N} u_{\varepsilon}^{*}(r)\right|^{2^{*}}\left(\left|B_{N} u_{\varepsilon}^{*}(r)\right|^{r^{\alpha}}-1\right) r^{N-1} d r .
$$

We estimate (setting $d_{N}:=\frac{A_{N}}{2^{(N-2) / 2}}$ )

$$
\begin{aligned}
I_{1, \varepsilon} & \geq \int_{0}^{\varepsilon}\left|u_{\varepsilon}(r)\right|^{2^{*}}\left[\left(\frac{A_{N} \varepsilon^{(N-2) / 2}}{\left(2 \varepsilon^{2}\right)^{(N-2) / 2}}\right)^{r^{\alpha}}-1\right] r^{N-1} d r \\
& \geq \int_{0}^{\varepsilon}\left|u_{\varepsilon}(r)\right|^{2^{*}}\left[\left(\frac{A_{N}}{2^{(N-2) / 2}}\right)^{r^{\alpha}} \varepsilon^{-\frac{N-2}{2} r^{\alpha}}-1\right] r^{N-1} d r \\
& =\int_{0}^{\varepsilon} \frac{A_{N}^{2^{*}} \varepsilon^{N}}{\left(2 \varepsilon^{2}\right)^{N}}\left(e^{r^{\alpha} \log d_{N}+\frac{N-2}{2} r^{\alpha}|\log \varepsilon|}-1\right) r^{N-1} d r \\
& \geq C \int_{0}^{\varepsilon} \frac{1}{\varepsilon^{N}}\left(r^{\alpha} \log d_{N}+\frac{N-2}{2} r^{\alpha}|\log \varepsilon|\right) r^{N-1} d r \\
& =C \frac{\log d_{N}+\frac{N-2}{2}|\log \varepsilon|}{\varepsilon^{N}} \int_{0}^{\varepsilon} r^{\alpha} r^{N-1} d r \\
& =\left.C \frac{\log d_{N}+\frac{N-2}{2}|\log \varepsilon|}{\varepsilon^{N}} \frac{1}{\alpha+N} r^{\alpha+N}\right|_{0} ^{\varepsilon} \\
& =C_{1}|\log \varepsilon| \varepsilon^{\alpha}+O\left(\varepsilon^{\alpha}\right) \\
& \geq C_{2}|\log \varepsilon| \varepsilon^{\alpha}, \text { for a suitable } C_{2}>0 \text { and } \varepsilon \text { sufficiently small, }
\end{aligned}
$$

which completes the proof.

\subsection{Normalized concentrating sequences.}

Definition 3.2. A sequence $\left(u_{n}\right) \subset H_{0, \text { rad }}^{1}(B)$ is a normalized concentrating sequence if

(i) $\left\|\nabla u_{n}\right\|_{L^{2}}=1$;

(ii) $u_{n} \rightarrow 0$ in $H_{0, \mathrm{rad}}^{1}(B)$;

(iii) for any $\delta>0: \int_{\delta}^{1}\left|u_{n}^{\prime}(r)\right|^{2} r^{N-1} d r \rightarrow 0$.

We denote by $\mathcal{N}$ the set of normalized concentrating sequences.

In the next proposition we characterize the maximal limit of the functional $\int_{0}^{1}|u|^{2^{*}+f(r)} r^{N-1} d x$ over $\mathcal{N}$. We restrict to the case that

$$
f(r)=r^{\alpha}, \alpha \in(0, \min \{N / 2, N-2\}) .
$$

\section{Proposition 3.3.}

$$
\sup _{\left(u_{n}\right) \in \mathcal{N}}\left\{\lim _{n \rightarrow \infty} \omega_{N-1} \int_{0}^{1}\left|u_{n}(r)\right|^{2^{*}+r^{\alpha}} r^{N-1} d r\right\} \leq \Sigma_{N} .
$$


Proof. It is sufficient to prove the following:

Given $\varepsilon>0$ there exists $\eta>0$ and $n_{0} \in \mathbb{N}$ such that for any $n \geq n_{0}$ we have

(a) $\quad \omega_{N-1} \int_{0}^{\eta}\left|u_{n}(r)\right|^{2^{*}+r^{\alpha}} r^{N-1} d r \leq \Sigma_{N}+\varepsilon / 2$;

(b) $\quad \omega_{N-1} \int_{\eta}^{1}\left|u_{n}(r)\right|^{2^{*}+r^{\alpha}} r^{N-1} d r \leq \varepsilon / 2$.

We first prove that (a) holds; indeed

$$
\begin{aligned}
& \quad \omega_{N-1} \int_{0}^{\eta}\left|u_{n}(r)\right|^{2^{*}+r^{\alpha}} r^{N-1} d r \\
& =\omega_{N-1} \int_{0}^{\eta}\left|u_{n}(r)\right|^{2^{*}} \\
& \left(\left|u_{n}(r)\right|^{r^{\alpha}}-1\right) r^{N-1} d r+\omega_{N-1} \int_{0}^{\eta}\left|u_{n}(r)\right|^{2^{*}} r^{N-1} d r \\
& \leq \omega_{N-1} \int_{0}^{\eta}\left|u_{n}(r)\right|^{2^{*}}\left(\left|u_{n}(r)\right|^{r^{\alpha}}-1\right) r^{N-1} d r+\Sigma_{N} .
\end{aligned}
$$

Using the Radial Lemma we can estimate

$$
\begin{aligned}
\int_{0}^{\eta}\left|u_{n}(r)\right|^{2^{*}} & \left(\left|u_{n}(r)\right|^{r^{\alpha}}-1\right) r^{N-1} d r \\
& \leq \int_{0}^{\eta}\left|u_{n}(r)\right|^{2^{*}}\left[\left(\frac{1}{r^{(N-2) / 2}}\right)^{r^{\alpha}}-1\right] r^{N-1} d r \\
& \leq \int_{0}^{\eta}\left|u_{n}(r)\right|^{2^{*}}\left[\exp \left(r^{\alpha} \log \left(\frac{1}{r^{(N-2) / 2}}\right)\right)-1\right] r^{N-1} d r \\
& \leq C \int_{0}^{\eta}\left|u_{n}(r)\right|^{2^{*}} r^{\alpha}\left|\log r^{(N-2) / 2}\right| r^{N-1} d r \\
& \leq C_{1} \eta^{\alpha}|\log \eta| \int_{0}^{1}\left|u_{n}(r)\right|^{2^{*}} r^{N-1} d r \\
& \leq C_{2} \eta^{\alpha}|\log \eta| \Sigma_{N} .
\end{aligned}
$$

Now taking $\eta=\eta(\varepsilon)>0$ sufficiently small such that $C_{2} \eta^{\alpha}|\log \eta| \Sigma_{N} \leq \varepsilon / 2$, we conclude that

$$
\int_{0}^{\eta}\left|u_{n}(r)\right|^{2^{*}}\left(\left|u_{n}(r)\right|^{r^{\alpha}}-1\right) r^{N-1} d r \leq \frac{\varepsilon}{2} .
$$

From (3.8) and (3.9) we obtain (a).

To prove (b) we proceed as follows: taking $t \in(\eta(\varepsilon), 1))$ we can write

$$
\begin{aligned}
\left|u_{n}(t)\right| & =\left|\int_{1}^{t} u_{n}^{\prime}(s) d s\right|=\left|\int_{1}^{t} u_{n}^{\prime}(s) s^{(N-1) / 2} \frac{1}{s^{(N-1) / 2}} d s\right| \\
& \leq\left.\left.\left|\int_{1}^{t}\right| u_{n}^{\prime}(s)\right|^{2} s^{N-1} d s\right|^{1 / 2}\left|\int_{1}^{t} \frac{1}{s^{N-1}} d s\right|^{1 / 2} \\
& \leq \sigma_{n}\left(t^{2-N}-1\right)^{1 / 2} \\
& \leq \sigma_{n} t^{\frac{2-N}{2}}
\end{aligned}
$$


where by assumption (iii)

$$
\sigma_{n}:=\left.\left.\left|\int_{\eta}^{1}\right| u_{n}^{\prime}(s)\right|^{2} s^{N-1} d s\right|^{1 / 2} \rightarrow 0 \quad \text { as } \quad n \rightarrow \infty .
$$

Then we can estimate

$$
\begin{aligned}
\int_{\eta}^{1}\left|u_{n}(r)\right|^{2^{*}+r^{\alpha}} r^{N-1} d x & \leq \int_{\eta}^{1}\left(\sigma_{n} r^{\frac{2-N}{2}}\right)^{2^{*}+r^{\alpha}} r^{N-1} d x \\
& \leq \sigma_{n}^{2^{*}} \int_{\eta}^{1}\left(r^{\frac{2-N}{2}}\right)^{2^{*}+r^{\alpha}} r^{N-1} d x \\
& \leq \sigma_{n}^{2^{*}} \int_{\eta}^{1} r^{-1-\frac{N-2}{2} r^{\alpha}} d x \\
& \leq \sigma_{n}^{2^{*}} c(\eta) \leq \frac{\varepsilon}{2}
\end{aligned}
$$

if we choose $n$ sufficiently large.

\section{Best Constant is attained}

We are now ready to prove that $\mathcal{U}_{N}$ is attained.

4.1. Proof of Theorem 1.4. Assume by contradiction that $\mathcal{U}_{N}$ is not attained. Let $\left(u_{n}\right)$ be a maximizing sequence. Since $\left(u_{n}\right)$ is bounded, there exists a weakly convergent subsequence $u_{n} \rightarrow w$, and hence by weak lower semicontinuity $\int_{B}|\nabla u|^{2} \leq 1$. We claim that $w=0$. If not, $\int_{B}|\nabla w|^{2}>0$.

By a Brezis-Lieb type argument we have (with $o(1) \rightarrow 0$ as $n \rightarrow \infty$ )

$$
\int_{B}\left|u_{n}(x)\right|^{2^{*}+r^{\alpha}} d x=\int_{B}\left|u_{n}(x)-w(x)\right|^{2^{*}+r^{\alpha}} d x+\int_{B}|w(x)|^{2^{*}+r^{\alpha}} d x+o(1)
$$

and

$$
1=\int_{B}\left|\nabla u_{n}(x)\right|^{2} d x=\int_{B}\left|\nabla\left(u_{n}(x)-w(x)\right)\right|^{2} d x+\int_{B}|\nabla w(x)|^{2} d x+o(1) .
$$

From the second identity follows that if $\int_{B}|\nabla w|^{2}=1$, then $u_{n} \rightarrow w$ strongly in $H_{0}^{1}(B)$ and hence $\mathcal{U}_{N}$ is attained by continuity, contradicting the assumption. Hence we can assume that $\int_{B}|\nabla w|^{2}<1$. 
Then, setting $z_{n}=u_{n}-w$, we have from $\int_{B}\left|u_{n}\right|^{2^{*}+r^{\alpha}} \rightarrow \mathcal{U}_{N}$ and the above identities that

$$
\begin{aligned}
\mathcal{U}_{N} & =\int_{B}\left|z_{n}\right|^{2^{*}+r^{\alpha}} d x+\int_{B}|w|^{2^{*}+r^{\alpha}} d x+o(1) \\
& =\int_{B}\left(\frac{\left|z_{n}\right|}{\left\|\nabla z_{n}\right\|_{2}}\right)^{2^{*}+r^{\alpha}}\left\|\nabla z_{n}\right\|_{2}^{2^{*}+r^{\alpha}} d x+\int_{B}\left(\frac{|w|}{\|\nabla w\|_{2}}\right)^{2^{*}+r^{\alpha}}\|\nabla w\|^{2^{*}+r^{\alpha}} d x+o(1) \\
& \leq \mathcal{U}_{N}\left\|\nabla z_{n}\right\|_{2}^{2^{*}}+\mathcal{U}_{N}\|\nabla w\|_{2}^{2^{*}}+o(1) \\
& =\mathcal{U}_{N}\left(\left(1-\|\nabla w\|_{2}^{2}+o(1)\right)^{2^{*} / 2}+\left(\|\nabla w\|_{2}^{2}\right)^{2^{*} / 2}\right)+o(1) \\
& <\mathcal{U}_{N} .
\end{aligned}
$$

This contradiction shows that $w=0$, and hence $u_{n} \rightarrow 0$.

We now show that $\left(u_{n}\right)$ is a normalized concentrating sequence. For this, we need to show that

$$
\int_{\delta}^{1}\left|u_{n}^{\prime}\right|^{2} r^{N-1} d r \rightarrow 0, \text { for any } \delta>0
$$

Recall that

$$
H_{\mathrm{rad}}^{1}([\delta, 1]) \subset \subset L^{p}([\delta, 1]), \forall p \geq 1
$$

and hence

$$
\int_{\delta}^{1}\left|u_{n}\right|^{2^{*}+r^{\alpha}} r^{N-1} d r \rightarrow 0
$$

Since $\left(u_{n}\right)$ is a maximizing sequence, we have by Ekeland's principle that there exists a multiplier $\lambda_{n}$ such that

$$
\lambda_{n} \int_{B} \nabla u_{n} \nabla \phi d x=\int_{B}\left(2^{*}+r^{\alpha}\right)\left|u_{n}\right|^{2^{*}-2+r^{\alpha}} u_{n} \phi d x+\langle o(1), \phi\rangle .
$$

Choosing $\phi=u_{n}$ we get

$$
\begin{aligned}
\lambda_{n} \int_{B}\left|\nabla u_{n}\right|^{2} d x & =\int_{B}\left(2^{*}+r^{\alpha}\right)\left|u_{n}\right|^{2^{*}+r^{\alpha}} d x+\left\langle o(1), u_{n}\right\rangle \\
& \geq 2^{*} \int_{B}\left|u_{n}\right|^{2^{*}+r^{\alpha}} d x \rightarrow 2^{*} \mathcal{U}_{N}
\end{aligned}
$$

and so we get $\liminf \operatorname{in}_{n \rightarrow \infty} \lambda_{n} \geq 2 * \mathcal{U}_{N}$.

Next, choose a smooth cut-off function

$$
\eta(r)= \begin{cases}0, & \text { if } r \leq \delta / 2 \\ 1, & \text { if } r \geq \delta\end{cases}
$$


and choose $\phi=\eta u_{n}$ in (4.3). Then we obtain by (4.2)

$$
\begin{array}{rl}
\int_{\delta / 2}^{1} u_{n}^{\prime}\left(\eta u_{n}\right)^{\prime} r^{N-1} & d r \\
& =\frac{1}{\lambda_{n}} \int_{\delta / 2}^{1}\left(2^{*}+r^{\alpha}\right)\left|u_{n}\right|^{2^{*}-2+r^{\alpha}} u_{n}\left(\eta u_{n}\right) r^{N-1} d r+\left\langle o(1), \eta u_{n}\right\rangle \rightarrow 0
\end{array}
$$

from which we get

$$
\begin{aligned}
o(1) & =\int_{\delta / 2}^{1} u_{n}^{\prime}\left(\eta u_{n}\right)^{\prime} r^{N-1} d r \\
& =\int_{\delta / 2}^{1} \eta\left|u_{n}^{\prime}\right|^{2} r^{N-1} d r+\int_{\delta / 2}^{1} u_{n}^{\prime} u_{n} \eta^{\prime} r^{N-1} d r \\
& \geq \int_{\delta}^{1}\left|u_{n}^{\prime}\right|^{2} r^{N-1} d r-\max \left|\eta^{\prime}\right|\left\|\nabla u_{n}\right\|_{2}\left\|u_{n}\right\|_{2} \\
& =\int_{\delta}^{1}\left|u_{n}^{\prime}\right|^{2} r^{N-1} d r+o(1),
\end{aligned}
$$

where we used that $\left\|u_{n}\right\|_{2} \rightarrow 0$ by the compact embedding. This proves (4.1).

Thus, we have shown that if $\mathcal{U}_{N}$ is not attained, then the maximizing sequence is a concentrating sequence, along which the functional tends to $\Sigma_{N}<\mathcal{U}_{N}$. This contradiction proves that $\mathcal{U}_{N}$ is attained.

\section{A SUPERCRitical EQUATION}

5.1. Proof of Theorem 1.5. We now consider the following highly supercritical equation (1.7). Indeed, note that the nonlinearity has critical Sobolev growth only in one point, namely in the origin, and is (even strongly) supercritical in all other points.

$$
\left\{\begin{aligned}
-\Delta u & =u^{2^{*}-1+r^{\alpha}} & & \text { in } B, \\
u & >0 & & \text { in } B, \\
u & =0 & & \text { on } \partial B,
\end{aligned}\right.
$$

To prove the existence of a solution, we employ variational methods. Consider the functional

$$
I(u)=\frac{1}{2} \int_{B}|\nabla u|^{2} d x-\int_{B} \frac{1}{2^{*}+r^{\alpha}}|u|^{2^{*}+r^{\alpha}} d x: H_{0, \text { rad }}^{1}(B) \rightarrow \mathbb{R} .
$$

Note that due to Theorem 1.1 the functional $I(u)$ is well-defined and of class $C^{1}$.

It is standard to see that the functional has a mountain-pass structure in the origin, but of course, due to the supercritical growth, the functional does not satisfy the Palais-Smale condition. To overcome this problem, we follow the strategy of the classical Brezis-Nirenberg paper: we identify the 
non-compactness level, and show that below this level there is compactness. Then, in a final step, we show that the minimax level of the functional lies indeed below the non-compactness level. We emphasize that this result does not require a perturbation with a lower order term: it is in fact the supercritical term which already guarantees this.

We proceed in three steps:

1) The level $\frac{1}{N} S_{N}^{\frac{N}{2}}$ is a noncompactness level for the functional $I(u)$.

2) The mountain-pass level $c$ of the functional $I(u)$ satisfies $c<\frac{1}{N} S_{N}^{\frac{N}{2}}$.

3) By 2) we obtain a weak solution $u$ at level $0<c<\frac{1}{N} S_{N}^{\frac{N}{2}}$. We show that then $u \neq 0$, which completes the proof.

In this section we denote again with

$$
\begin{aligned}
u_{\varepsilon}^{*}(r) & =C_{N} \frac{\varepsilon^{\frac{N-2}{2}}}{\left(\varepsilon^{2}+r^{2}\right)^{\frac{N-2}{2}}}, \quad \varepsilon>0 \\
C_{N} & :=(N(N-2))^{\frac{N-2}{2}}
\end{aligned}
$$

the standard Sobolev instantons, which satisfy the equation

$$
-\Delta u=u^{2^{*}-1}, \text { on } \mathbb{R}^{N}
$$

and for which

$$
\int_{\mathbb{R}^{N}}\left|\nabla u_{\varepsilon}^{*}\right|^{2} d x=S_{N}^{N / 2} \text { and } \int_{\mathbb{R}^{N}}\left|u_{\varepsilon}^{*}\right|^{2^{*}} d x=S_{N}^{N / 2}
$$

1) Taking a suitable cut-off function $\eta$ and setting $u_{\varepsilon}=\eta u_{\varepsilon}^{*}$ one checks that $u_{\varepsilon} \in H_{0, \text { rad }}^{1}(B)$ is a Palais-Smale sequence, with

$$
I\left(u_{\varepsilon}\right)=\frac{1}{2} \int_{B}\left|\nabla u_{\varepsilon}\right|^{2} d x-\int_{B} \frac{1}{2^{*}+r^{\alpha}}\left|u_{\varepsilon}\right|^{2^{*}+r^{\alpha}} d x \rightarrow \frac{1}{N} S_{N}^{N / 2} .
$$

The sequence $u_{\varepsilon}$ is concentrating and converges weakly to 0 , and thus it does not contain a strongly convergent subsequence; hence we have proved 1).

2) It is clear that the functional $I$ has a Mountain-Pass structure. To prove that the moutain-pass level lies below the value $\frac{1}{N} S_{N}^{\frac{N}{2}}$, we choose $u_{\varepsilon}$ as in 1), and set

$$
c=\inf _{\gamma \in \Gamma} \max _{u \in \gamma} I(u)
$$

where

$$
\Gamma:=\left\{\gamma:[0, R] \rightarrow H_{0}^{1}(B) \text { continuous }, \gamma(0)=0, \gamma(R)=R u_{\varepsilon}\right\}
$$


with $R>0$ sufficiently large, so that $I\left(R u_{\varepsilon}\right) \leq 0$. Then the path $\gamma_{\varepsilon}(t)=$ $t u_{\varepsilon}, t \in[0, R]$, belongs to $\Gamma$, and

$$
c \leq \max _{t \in[0, R]} I\left(t u_{\varepsilon}\right)=: I\left(t_{\varepsilon} u_{\varepsilon}\right)
$$

We first estimate the value of $t_{\varepsilon}$, using similar estimates as in the proof of Proposition 3.3. Since $\left.\frac{d}{d t} I\left(t u_{\varepsilon}\right)\right|_{t=t_{\varepsilon}}=0$, we get

$$
t_{\varepsilon} \int_{B}\left|\nabla u_{\varepsilon}(x)\right|^{2} d x=\int_{B} t_{\varepsilon}^{2^{*}+r^{\alpha}-1}\left|u_{\varepsilon}(x)\right|^{2^{*}+r^{\alpha}} d x
$$

It is known (see [2]) that

$$
\int_{B}\left|\nabla u_{\varepsilon}(x)\right|^{2} d x=S_{N}^{\frac{N}{2}}+O\left(\varepsilon^{N-2}\right), \int_{B}\left|u_{\varepsilon}(x)\right|^{2^{*}} d x=S_{N}^{\frac{N}{2}}+O\left(\varepsilon^{N}\right) .
$$

Hence we get from (5.5)

$$
\begin{aligned}
S_{N}^{\frac{N}{2}}+O\left(\varepsilon^{N-2}\right) & =t_{\varepsilon}^{2^{*}-2} \int_{B}\left|u_{\varepsilon}(x)\right|^{2^{*}} d x+t_{\varepsilon}^{2^{*}-2} \int_{B}\left(t_{\varepsilon}^{r^{\alpha}}\left|u_{\varepsilon}(x)\right|^{2^{*}+r^{\alpha}}-\left|u_{\varepsilon}(x)\right|^{2^{*}}\right) d x \\
& =t_{\varepsilon}^{2^{*}-2}\left[S_{N}^{\frac{N}{2}}+O\left(\varepsilon^{N}\right)+\int_{B}\left|u_{\varepsilon}(x)\right|^{2^{*}}\left(\left(t_{\varepsilon}\left|u_{\varepsilon}(x)\right|\right)^{r^{\alpha}}-1\right) d x\right] \\
& =t_{\varepsilon}^{2^{*}-2}\left[S_{N}^{\frac{N}{2}}+O\left(\varepsilon^{N}\right)+A_{\varepsilon}\right]
\end{aligned}
$$

Estimate of $A_{\varepsilon}$ : let $\tilde{a}_{\varepsilon}$ such that $\left|t_{\varepsilon} u_{\varepsilon}\right| \leq 1$ for $r \geq \tilde{a}_{\varepsilon}$ (recall that by the mountain-pass structure one has $\delta \leq t_{\varepsilon} \leq R$ )

$$
\begin{aligned}
A_{\varepsilon}= & \int_{B}\left|u_{\varepsilon}(x)\right|^{2^{*}}\left(\left|t_{\varepsilon} u_{\varepsilon}(x)\right|^{r^{\alpha}}-1\right) d x \\
& \leq \omega_{N-1} \int_{0}^{\tilde{a}_{\varepsilon}}\left|u_{\varepsilon}(r)\right|^{2^{*}}\left(\left|t_{\varepsilon} u_{\varepsilon}(r)\right|^{r^{\alpha}}-1\right) r^{N-1} d r \\
& =\omega_{N-1} \int_{0}^{\tilde{a}_{\varepsilon}}\left|u_{\varepsilon}(r)\right|^{2^{*}}\left(e^{r^{\alpha} \log \left|t_{\varepsilon} u_{\varepsilon}(r)\right|}-1\right) r^{N-1} d r \\
& \leq c \int_{0}^{\varepsilon}\left|u_{\varepsilon}(r)\right|^{2^{*}} r^{\alpha}|\log \varepsilon| r^{N-1} d r+c \int_{\varepsilon}^{\tilde{a}_{\varepsilon}}\left|u_{\varepsilon}(r)\right|^{2^{*}} r^{\alpha}|\log \varepsilon| r^{N-1} d r \\
& \leq c \int_{0}^{\varepsilon} \varepsilon^{-N} r^{\alpha}|\log \varepsilon| r^{N-1} d r+c \int_{\varepsilon}^{\tilde{a}_{\varepsilon}} \varepsilon^{N} r^{-2 N} r^{\alpha}|\log \varepsilon| r^{N-1} d r \\
& \leq c \varepsilon^{\alpha}|\log \varepsilon|+\left.c \varepsilon^{N}|\log \varepsilon|\left(-r^{-N+\alpha}\right)\right|_{\varepsilon} ^{\tilde{a}_{\varepsilon}} \\
& \leq c \varepsilon^{\alpha}|\log \varepsilon|
\end{aligned}
$$


and

$$
\begin{aligned}
A_{\varepsilon} & \geq \omega_{N-1} \int_{\tilde{a}_{\varepsilon}}^{1}\left|u_{\varepsilon}(r)\right|^{2^{*}}\left(\left|t_{\varepsilon} u_{\varepsilon}(r)\right|^{r^{\alpha}}-1\right) r^{N-1} d r \\
& \geq-\omega_{N-1} \int_{\tilde{a}_{\varepsilon}}^{1}\left|u_{\varepsilon}(r)\right|^{2^{*}} r^{N-1} d r \\
& \geq-c \int_{\tilde{a}_{\varepsilon}}^{1} \frac{\varepsilon^{N}}{r^{2 N}} r^{N-1} d r \\
& =\left.c \varepsilon^{N} r^{-N}\right|_{\tilde{a}_{\varepsilon}} ^{1} \\
& \geq-c \varepsilon^{N / 2} .
\end{aligned}
$$

Thus, we obtain

$$
S_{N}^{\frac{N}{2}}+O\left(\varepsilon^{N-2}\right)=t_{\varepsilon}^{2^{*}-2}\left[S^{\frac{N}{2}}+O\left(\varepsilon^{N}\right)+O\left(\varepsilon^{\alpha}|\log \varepsilon|\right)+O\left(\varepsilon^{\frac{N}{2}}\right)\right]
$$

from which we see that $t_{\varepsilon} \rightarrow 1$, and then we get

$$
1+O\left(\varepsilon^{N-2}\right)=\left(1+\left(2^{*}-2\right)\left(t_{\varepsilon}-1\right)\right)\left[1+O\left(\varepsilon^{\alpha}|\log \varepsilon|\right)+O\left(\varepsilon^{\frac{N}{2}}\right)\right]
$$

from which we finally obtain, assuming that $0<\alpha<\min \left\{N-2, \frac{N}{2}\right\}$

$$
\begin{aligned}
t_{\varepsilon}-1 & =O\left(\varepsilon^{N-2}\right)+O\left(\varepsilon^{\alpha}|\log \varepsilon|\right)+O\left(\varepsilon^{\frac{N}{2}}\right) \\
& =O\left(\varepsilon^{\alpha}|\log \varepsilon|\right)=: R_{\varepsilon} .
\end{aligned}
$$


We now return to the estimate of the level $c$, see (5.4): we have

$$
\begin{aligned}
I\left(t_{\varepsilon} u_{\varepsilon}\right)= & \frac{1}{2} \int_{B} t_{\varepsilon}^{2}\left|\nabla u_{\varepsilon}\right|^{2}-\int_{B} \frac{t_{\varepsilon}^{2^{*}+r^{\alpha}}}{2^{*}+r^{\alpha}}\left|u_{\varepsilon}\right|^{2^{*}+r^{\alpha}} \\
= & \frac{1}{2}\left(1+R_{\varepsilon}\right)^{2}\left(S_{N}^{\frac{N}{2}}+O\left(\varepsilon^{N-2}\right)\right)-\int_{B} \frac{\left(1+R_{\varepsilon}\right)^{2^{*}+r^{\alpha}}}{2^{*}+r^{\alpha}}\left|u_{\varepsilon}\right|^{2^{*}+r^{\alpha}} \\
= & \frac{1}{2} S_{N}^{\frac{N}{2}}+R_{\varepsilon} S_{N}^{\frac{N}{2}}+c R_{\varepsilon}^{2}+O\left(\varepsilon^{N-2}\right)-\int_{B} \frac{\left(1+R_{\varepsilon}\right)^{2^{*}+r^{\alpha}}}{2^{*}+r^{\alpha}}\left|u_{\varepsilon}\right|^{2^{*}} \\
& \quad-\int_{B} \frac{\left(1+R_{\varepsilon}\right)^{2^{*}+r^{\alpha}}}{2^{*}+r^{\alpha}}\left(\left|u_{\varepsilon}\right|^{2^{*}+r^{\alpha}}-\left|u_{\varepsilon}\right|^{2^{*}}\right) \\
\leq & \frac{1}{2} S_{N}^{\frac{N}{2}}+R_{\varepsilon} S_{N}^{\frac{N}{2}}+c R_{\varepsilon}^{2}+O\left(\varepsilon^{N-2}\right)-\int_{B} \frac{1+\left(2^{*}+r^{\alpha}\right) R_{\varepsilon}+c R_{\varepsilon}^{2}}{2^{*}+r^{\alpha}}\left|u_{\varepsilon}\right|^{2^{*}} \\
& -d \int_{B}\left(\left|u_{\varepsilon}\right|^{2^{*}+r^{\alpha}}-\left|u_{\varepsilon}\right|^{2^{*}}\right) \\
\leq & \frac{1}{2} S_{N}^{\frac{N}{2}}+R_{\varepsilon} S_{N}^{\frac{N}{2}}+c R_{\varepsilon}^{2}+O\left(\varepsilon^{N-2}\right)-\int_{B} \frac{1}{2^{*}+r^{\alpha}}\left|u_{\varepsilon}\right|^{2^{*}}-R_{\varepsilon} S_{N}^{\frac{N}{2}} \\
& +O\left(R_{\varepsilon} \varepsilon^{N}\right)+O\left(R_{\varepsilon}^{2}\right)-d \int_{B}\left(\left|u_{\varepsilon}\right|^{2^{*}+r^{\alpha}}-\left|u_{\varepsilon}\right|^{2^{*}}\right) \\
= & \frac{1}{2} S_{N}^{\frac{N}{2}}+O\left(R_{\varepsilon}^{2}\right)+O\left(\varepsilon^{N-2}\right)-\frac{1}{2^{*}} S_{N}^{\frac{N}{2}}+O\left(\varepsilon^{N}\right)+\int_{B}\left(\frac{1}{2^{*}}-\frac{1}{2^{*}+r^{\alpha}}\right)\left|u_{\varepsilon}\right|^{2^{*}} \\
& -d \int_{B}\left(\left|u_{\varepsilon}\right|^{2^{*}+r^{\alpha}}-\left|u_{\varepsilon}\right|^{2^{*}}\right) \\
= & \frac{1}{N} S_{N}^{\frac{N}{2}}+O\left(R_{\varepsilon}^{2}\right)+O\left(\varepsilon^{N-2}\right)+c \varepsilon^{\alpha}-c \varepsilon^{\alpha}|\log \varepsilon| \\
S_{N}^{\frac{N}{2}} & \text { for } \varepsilon>0 \text { sufficiently small },
\end{aligned}
$$

where we have used that

$$
\begin{gathered}
\int_{B}\left(\frac{1}{2^{*}}-\frac{1}{2^{*}+r^{\alpha}}\right)\left|u_{\varepsilon}\right|^{2^{*}} d x \leq c \int_{B} r^{\alpha}\left|u_{\varepsilon}\right|^{2^{*}} r^{N-1} d r \\
\quad \leq c \int_{0}^{\varepsilon} r^{\alpha} \varepsilon^{-N} r^{N-1} d r+c \int_{\varepsilon}^{1} r^{\alpha} \frac{\varepsilon^{N}}{r^{2 N}} r^{N-1} d r \\
\quad \leq c \varepsilon^{\alpha}+c\left(\varepsilon^{\alpha}-\varepsilon^{N}\right)=c \varepsilon^{\alpha}
\end{gathered}
$$


and

$$
\begin{aligned}
\int_{B} & \left(\left|u_{\varepsilon}(x)\right|^{2^{*}+r^{\alpha}}-\left|u_{\varepsilon}(x)\right|^{2^{*}}\right) d r=\int_{B}\left|u_{\varepsilon}(x)\right|^{2^{*}}\left(\left|u_{\varepsilon}(x)\right|^{r^{\alpha}}-1\right) d r \\
& \geq \int_{0}^{\varepsilon}\left|u_{\varepsilon}(r)\right|^{2^{*}}\left(\left|u_{\varepsilon}(r)\right|^{r^{\alpha}}-1\right) r^{N-1} d r-\int_{a_{\varepsilon}}^{1}\left|u_{\varepsilon}(r)\right|^{2^{*}}\left(1-\left|u_{\varepsilon}(r)\right|^{r^{\alpha}}\right) r^{N-1} d r \\
& \geq \int_{0}^{\varepsilon} \varepsilon^{-N} r^{\alpha}|\log \varepsilon| r^{N-1} d r-\int_{a_{\varepsilon}}^{1}\left|u_{\varepsilon}\right|^{2^{*}} r^{N-1} d r \\
& \geq \varepsilon^{\alpha}|\log \varepsilon|-\varepsilon^{N} \int_{a_{\varepsilon}}^{1} r^{-2 N} r^{N-1} d r \\
& \geq \varepsilon^{\alpha}|\log \varepsilon|-\varepsilon^{N}\left(\varepsilon^{-N / 2}-1\right) \\
& \geq \varepsilon^{\alpha}|\log \varepsilon| .
\end{aligned}
$$

3) By 2) we obtain a $(P S)_{c}$ sequence $\left(u_{n}\right)$ with

$$
I\left(u_{n}\right) \rightarrow c<\frac{1}{N} S_{N}^{\frac{N}{2}}
$$

and

$$
I^{\prime}\left(u_{n}\right)[\varphi]=\omega_{N-1} \int_{0}^{1} u_{n}^{\prime} \varphi^{\prime} r^{N-1} d r-\omega_{N-1} \int_{0}^{1}\left|u_{n}\right|^{2^{*}-1+r^{\alpha}} \varphi r^{N-1} d r \rightarrow 0 .
$$

It is standard to show that $\left\{u_{n}\right\} \subset H_{0}^{1}(\Omega)$ is bounded, and hence there is a weakly convergent subsequence

$$
u_{n} \rightarrow u, n \rightarrow \infty
$$

which solves weakly equation (5.3). If $u \neq 0$ we are done. Hence we assume that $u=0$, and show that this is impossible.

As in (4.2) we have

$$
\int_{\delta}^{1}\left|u_{n}(r)\right|^{2^{*}+r^{\alpha}} r^{N-1} d r \rightarrow 0, \text { for } \delta>0 \text { fixed. }
$$

Taking $\eta$ as in (4.4) and choosing $\varphi=\eta u_{n}$ in (5.6) we obtain

$$
\int_{\delta / 2}^{1} u_{n}^{\prime}\left(\eta u_{n}\right)^{\prime} r^{N-1} d r=\int_{\delta / 2}^{1}\left|u_{n}\right|^{2^{*}-2+r^{\alpha}}\left(\eta u_{n}\right) r^{N-1} d r+\left\langle o(1), \eta u_{n}\right\rangle \rightarrow 0
$$

from which we get as in (4.5) that

$$
\int_{\delta}^{1}\left|u_{n}^{\prime}(r)\right|^{2} r^{N-1} d r \rightarrow 0, \text { for any } \delta>0
$$

Next, we show that

$$
I\left(u_{n}\right)=I_{0}\left(u_{n}\right)+o(1)
$$


where

$$
I_{0}(w)=\frac{1}{2} \int_{B}|\nabla w(x)|^{2} d x-\frac{1}{2^{*}} \int_{B}|w(x)|^{2^{*}} d x .
$$

Indeed, we have

$$
\begin{aligned}
& \int_{0}^{1}\left|u_{n}\right|^{2^{*}+r^{\alpha}} r^{N-1} d r=\int_{0}^{1}\left|u_{n}\right|^{2^{*}} r^{N-1} d r+\int_{0}^{1}\left(\left|u_{n}\right|^{2^{*}+r^{\alpha}} d x-\left|u_{n}\right|^{2^{*}}\right) r^{N-1} d r \\
& =\int_{0}^{1}\left|u_{n}\right|^{2^{*}} r^{N-1} d r+\int_{0}^{\eta}\left|u_{n}\right|^{2^{*}}\left(\left|u_{n}\right|^{r^{\alpha}}-1\right) r^{N-1} d r+\int_{\eta}^{1}\left|u_{n}\right|^{2^{*}}\left(\left|u_{n}\right|^{r^{\alpha}}-1\right) r^{N-1} d r \\
& \leq \int_{0}^{1}\left|u_{n}\right|^{2^{*}} r^{N-1} d r+\frac{\varepsilon}{2}+\int_{\eta}^{1}\left|u_{n}\right|^{2^{*}}\left|u_{n}\right|^{r^{\alpha}} r^{N-1} d r \\
& \leq \int_{0}^{1}\left|u_{n}\right|^{2^{*}} r^{N-1} d r+\varepsilon
\end{aligned}
$$

by (3.9) and (4.2).

Similarly, one shows that

$$
I^{\prime}\left(u_{n}\right)[\varphi]=I_{0}^{\prime}\left(u_{n}\right)[\varphi]+o(1)
$$

Hence, we obtain that $\left(u_{n}\right)$ is a $(P S)_{c}$ sequence also for the functinal $I_{0}$. However, it is known that for $I_{0}$ the Palais-Smale condition holds for $0<c<$ $\frac{1}{N} S_{N}^{\frac{N}{2}}$, and hence, for a subsequence, we have that $u_{n} \rightarrow u=0$ strongly in $H_{0}^{1}(\Omega)$. But this implies that $I\left(u_{n}\right) \rightarrow 0$, in contradiction to $I\left(u_{n}\right) \rightarrow c>0$.

\section{REFERENCES}

[1] Bahri, A.; Coron, J.-M., On a nonlinear elliptic equation involving the critical Sobolev exponent: the effect of the topology of the domain, Comm. Pure Appl. Math. 41 (1988), no. 3

[2] Brezis,H., Nirenberg, L., Positive solutions of nonlinear elliptic equations involving critical sobolev exponents, Comm. Pure Appl. Math. 36 (1983) 437-477.

[3] Coron, J.M., Topologie et cas limite des injections de Sobolev, C. R. Acad. Sci. Paris Ser. I 299 (1984) 209-212.

[4] del Pino, M., Nonlinear elliptic problems above criticality, Milan J. Math. 74 (2006) 313-338.

[5] del Pino, M., Supercritical elliptic problems from a perturbation viewpoint, Discrete Contin. Dyn. Syst. 21 (2008), no. 1, 69-89.

[6] del Pino, M., Felmer, P., Musso, M., Multi-peak solution for super-critical elliptic problems in domains with small holes, J. Differential Equations 182 (2002) 511-540.

[7] del Pino, M., Wei, J., Supercritical elliptic problems in domains with small holes, Ann. Inst. H. Poincar Anal. Non Lineaire 24 (2007) 507-520

[8] Diening, L., Harjulehto, P., Hst, P., Ruzicka, M., Lebesgue and Sobolev Spaces with Variable Exponents, Lecture Notes in Mathematics 2017, Springer (2011)

[9] Peetre, J., Espaces dinterpolation et thorme de Soboleff, Ann. Inst. Fourier 16, 279-317 (1966)

[10] Pohozaev, S., Eigenfunctions of the equation $u+f(u)=0$, Soviet Math. Doklady 6 (1965), 1408-1411. 
[11] Ruf, B., Srikanth, P.N., Singularly perturbed elliptic equations with solutions concentrating on a 1-dimensional orbit, J. Eur. Math. Soc. (JEMS) 12 (2010), no. 2, 413-427.

[12] Ruf, B., Srikanth, P. N., Concentration on Hopf-fibres for singularly perturbed elliptic equations, J. Funct. Anal. 267 (2014), no. 7, 2353-2370.

[13] Struwe, M., Variational Methods. Applications to Nonlinear Partial Differential Equations and Hamiltonian Systems, Springer, 1990.

[14] Talenti, G., Best constant in Sobolev inequality, Ann. Mat. Pura Appl. (4) 110 (1976), 353372 .

[15] Tartar, L., Imbedding theorems of Sobolev spaces into Lorentz spaces, Boll. Unione Mat. Ital. Sez. B Artic. Ric. Mat. 8(1), 479-500 (1998)

[16] W. A. Strauss, Existence of solitary waves in higher dimensions, Comm. Math. Phys. 55 (1977), 149-162.

(J.M. do Ó) Dep. Mathematics, Federal University of Paraíba 58051-900, Jỗo PEssoA-PB, BRAZIL

E-mail address: jmbo@pq.cnpq.br

(B. Ruf) Dip. Matematica, Università di Milano

Via Saldini 50, 20133 Milano, Italy

E-mail address: bernhard.ruf@unimi.it

(P. Ubilla) Dep. Matemáticas y C.C., Universidad de Santiago de Chile

Casilla 307, Correo 2, Santiago - Chile

E-mail address: pubilla.ubilla@usach.cl 